This item was submitted to Loughborough's Research Repository by the author.

Items in Figshare are protected by copyright, with all rights reserved, unless otherwise indicated.

\title{
On single-stage DEA models with weight restrictions
}

PLEASE CITE THE PUBLISHED VERSION

http://dx.doi.org/10.1016/j.ejor.2015.07.050

\section{PUBLISHER}

(C) Elsevier and Association of European Operational Research Societies (EURO) within the International Federation of Operational Research Societies (IFORS)

\section{VERSION}

AM (Accepted Manuscript)

\section{PUBLISHER STATEMENT}

This work is made available according to the conditions of the Creative Commons Attribution-NonCommercialNoDerivatives 4.0 International (CC BY-NC-ND 4.0) licence. Full details of this licence are available at: https://creativecommons.org/licenses/by-nc-nd/4.0/

\section{LICENCE}

CC BY-NC-ND 4.0

\section{REPOSITORY RECORD}

Podinovski, Victor V., and Tatiana Bouzdine-Chameeva. 2019. "On Single-stage DEA Models with Weight Restrictions". figshare. https://hdl.handle.net/2134/18722. 


\title{
On single-stage DEA models with weight restrictions
}

\section{Victor V. Podinovski}

School of Business and Economics, Loughborough University, Loughborough LE11 3TU, UK

e-mail: v.podinovski@lboro.ac.uk

Phone: +44 1509228377

\section{Tatiana Bouzdine-Chameeva}

KEDGE Business School, 680 cours de la Libération, 33405 Talence Cedex, France

\begin{abstract}
The literature on data envelopment analysis (DEA) often employs multiplier models that incorporate very small (theoretically infinitesimal) lower bounds on the input and output weights. Computational problems arising from the solution of such programs are well known. In this paper we identify an additional theoretical problem that may arise if such bounds are used in a multiplier model with weight restrictions. Namely, we show that the use of small lower bounds may lead to the identification of an efficient target with negative inputs. We suggest a corrected model that overcomes this problem.
\end{abstract}

Keywords: Data envelopment analysis, weight restrictions, production trade-offs 


\section{Introduction}

In standard models of data envelopment analysis (DEA), the radial input and output projections of decision making units (DMUs) often exhibit mix inefficiency (are only weakly but not strongly efficient) - see, e.g., Cooper et al. (2007) and Thanassoulis et al. (2008). Such projected DMUs cannot therefore be generally regarded as efficient targets. A common approach to the identification of efficient targets is the maximisation of component (input and output) slacks as a secondary optimisation goal. There are two principal approaches known in the literature that allow us to implement this procedure: the optimisation of the radial improvement factor and component slacks can be performed either in a single optimisation stage or in separate stages.

In the single-stage approach, the multiplier DEA model is solved by incorporating a small (theoretically infinitesimal) lower bound $\varepsilon>0$ on all input and output weights. Equivalently, the objective function of the dual envelopment model is modified to include the input and output slacks multiplied by $\varepsilon$. The purpose of this approach is to let the DEA model assess the input or output radial efficiency of a DMU, while simultaneously accounting for possible mix inefficiency as a secondary goal (hence the requirement that $\varepsilon$ be very small).

Ali and Seiford (1993a) point out that the single-stage approach may lead to computational problems. In order to keep the assessment of radial efficiency as the main goal and mix improvements as the secondary objective, $\varepsilon>0$ needs to be as small as possible. However, the use of a very small $\varepsilon$ may lead to unacceptable inaccuracies arising from the finite precision of computer solutions. Because of this drawback, the two-stage optimisation approach of Ali and Seiford (1993b) appears to have become a preferred solution method of DEA models. ${ }^{1}$

In this paper we consider DEA models with weight restrictions. These are additional constraints often imposed on variable input and/or output weights in multiplier DEA models (Thanassoulis et al., 2004; Podinovski, 1999, 2004b; Cook and Zhu, 2008). Such restrictions may be used to incorporate value judgements in a DEA model and generally lead to an improved ability of the model to discriminate between the efficiency of DMUs (Allen et al., 1997).

\footnotetext{
${ }^{1}$ Another problem identified by Ali and Seiford (1993a) is that for insufficiently small values of $\varepsilon$ the multiplier model may be infeasible and its dual envelopment model unbounded. This issue has attracted further research in the literature but is unrelated to our results.
} 
The incorporation of weight restrictions in a multiplier DEA model generates additional terms in the dual envelopment model. Podinovski (2004a) shows that such terms are interpretable as production trade-offs - the latter express simultaneous changes to the inputs and outputs deemed possible if implemented at any DMU in the technology. This interpretation explains an earlier observation (Charnes et al., 1989, Roll et al., 1991) that the use of weight restrictions leads to an expansion of the model of technology.

The dual relationship between weight restrictions and production trade-offs can be used as a basis for the construction of weight restrictions for which the expanded technology and its efficient frontier are technologically meaningful (i.e., all DMUs in the expanded technology are technologically feasible, or producible). For example, Podinovski (2007a) assesses production trade-offs and the corresponding weight restrictions in the context of higher education. Amado and Dyson (2009) and Amado and Santos (2009) use this method in applications of DEA to health care provision. Santos et al. (2011) and Santos and Amado (2014) construct production trade-offs and weight restrictions for electricity distribution utilities and the court system. Atici and Podinovski (2015) illustrate the use of trade-offs in the assessment of efficiency of agricultural farms.

It may appear that the two solution methods applicable to DEA models without weight restrictions should also be applicable to models with weight restrictions. However, as shown by Podinovski (2007b), if the standard second optimisation stage is used to solve DEA models with weight restrictions, the resulting target DMU may have negative inputs, which is often meaningless. This problem is overcome by the modified second optimisation stage suggested by Podinovski (2007b).

In this paper we consider the use of the standard single-stage procedure for DEA models with weight restrictions and show its potential shortcomings. Our development is motivated by a significant DEA literature in which weight restrictions of different types are incorporated in the multiplier models together with the lower bound $\varepsilon>0$ on all input and output weights.

We give examples of efficiency assessment in which the use of an arbitrarily small lower bound $\varepsilon>0$ on the multiplier weights results in a negative input of the efficient target DMU. This problem occurs under both assumptions of constant (CRS) and variable (VRS) returns to scale (Charnes et al., 1978; Banker et al., 1984). We show that this effect arises not because of computational inaccuracies (our solutions are exact) but simply as a consequence of accounting for the radial and mix inefficiency in the same optimisation stage. Therefore, 
even disregarding computational problems, a straightforward incorporation of the lower multiplier bound $\varepsilon>0$ is theoretically unsound.

To overcome the noted problem, we suggest a modification to the single-stage formulation. This makes the new single-stage model (in both multiplier and envelopment forms) theoretically correct and useful for the compact expression of the two objectives: the assessment of radial and mix inefficiency of a DMU. We show that the corrected single-stage model may also be useful for the exact solution of simple illustrative examples.

The suggested model does not address the computational difficulties discussed by Ali and Seiford (1993a). In practical computations, preference should still be given to the use of the two-stage method of Ali and Seiford (1993b). For models with weight restrictions, the second stage of this method needs a modification developed by Podinovski (2007b).

\section{Production technology with weight restrictions}

In this section we briefly outline the dual relationship between weight restrictions and production trade-offs. We also clarify the notion of production technology arising in DEA models with weight restrictions. ${ }^{2}$ We use these notions in Section 3 to show that the singlestage statement of DEA models may lead to an incorrect identification of efficient targets.

To be specific, consider assessing the output radial efficiency of DMU $\left(X_{o}, Y_{o}\right)$ by a CRS multiplier model that incorporates the following $K$ weight restrictions on the vectors $v$ and $u$ of input and output weights, respectively:

$$
v^{\top} P_{t}-u^{\top} Q_{t} \geq 0, \quad t=1, \ldots, K .
$$

We refer to inequalities (1) with zero on the right-hand side as homogeneous weight restrictions (Podinovski 2004a). ${ }^{3}$ Components of vectors $P_{t} \in \mathbb{R}^{m}$ and $Q_{t} \in \mathbb{R}^{s}$ may be positive, negative or zero.

Consider the standard CRS output-oriented multiplier model that incorporates weight restrictions (1). Let $X_{j} \in \mathbb{R}_{+}^{m}$ and $Y_{j} \in \mathbb{R}_{+}^{s}$ denote the input and output vectors of observed

\footnotetext{
${ }^{2}$ For a theoretical development of this production technology, see Podinovski (2004a, 2015) and Podinovski and Bouzdine-Chameeva (2013, 2015).

${ }^{3}$ Non-homogeneous weight restrictions have a non-zero constant on the right-hand side. Examples of such weight restrictions are absolute weight bounds (Dyson and Thanassoulis, 1988). Using the normalising equality of the multiplier model (2), or its input-oriented analogue, non-homogeneous weight restrictions can be restated as homogeneous ones. However, the latter will depend on the unit under the assessment and the orientation of the model. An extended discussion of non-homogeneous weight restrictions is presented in Podinovski (2005).
} 
DMUs $j=1, \ldots, n$. The output radial efficiency of DMU $\left(X_{o}, Y_{o}\right)$ is the inverse of the optimal value $\eta^{*}$ of the following linear program:

$$
\eta^{*}=\min \quad v^{\top} X_{o}
$$

subject to $\quad u^{\top} Y_{o}=1$,

$$
\begin{array}{ll}
v^{\top} X_{j}-u^{\top} Y_{j} \geq 0, & j=1, \ldots, n, \\
v^{\top} P_{t}-u^{\top} Q_{t} \geq 0, & t=1, \ldots, K, \\
u, v \geq 0 . &
\end{array}
$$

The envelopment model dual to (2) can be stated as follows (the constraints of the dual are stated as equalities by using nonnegative slack vectors $e$ and $d$ ):

$$
\begin{array}{ll}
\eta^{*}=\max & \eta, \\
\text { subject to } & \sum_{j=1}^{n} \lambda_{j} X_{j}+\sum_{t=1}^{K} \pi_{t} P_{t}+d=X_{o}, \\
& \sum_{j=1}^{n} \lambda_{j} Y_{j}+\sum_{t=1}^{K} \pi_{t} Q_{t}-e=\eta Y_{o}, \\
& \lambda, \pi, d, e \geq 0, \eta \text { sign free. }
\end{array}
$$

Observe that the incorporation of weight restrictions (1) in the multiplier model (2) has generated additional dual terms

$$
\left(P_{t}, Q_{t}\right), \quad t=1, \ldots, K,
$$

in the envelopment model (3) applied in variable proportions $\pi_{t} \geq 0$. Podinovski (2004a) refers to these terms as production trade-offs. The envelopment model (3) projects DMU $\left(X_{o}, Y_{o}\right)$ on the boundary of the expanded CRS technology defined as follows.

Definition 1 (Podinovski 2004a). The CRS technology with production trade-offs $\mathcal{T}_{\text {CRS-TO }}$ is the set of all DMUs $(X, Y) \in \mathbb{R}_{+}^{m} \times \mathbb{R}_{+}^{s}$ for which there exist intensity vectors $\lambda \in \mathbb{R}_{+}^{n}$, $\pi \in \mathbb{R}_{+}^{K}$, and slack vectors $d \in \mathbb{R}_{+}^{m}$ and $e \in \mathbb{R}_{+}^{s}$ such that

$$
\begin{aligned}
& \sum_{j=1}^{n} \lambda_{j} X_{j}+\sum_{t=1}^{K} \pi_{t} P_{t}+d=X, \\
& \sum_{j=1}^{n} \lambda_{j} Y_{j}+\sum_{t=1}^{K} \pi_{t} Q_{t}-e=Y .
\end{aligned}
$$


The meaning of Definition 1 is straightforward. The terms $\sum_{j=1}^{n} \lambda_{j} X_{j}$ and $\sum_{j=1}^{n} \lambda_{j} Y_{j}$ on the left-hand side of equations (4) describe an arbitrary DMU in the standard CRS technology. The terms $\sum_{t=1}^{K} \pi_{t} P_{t}$ and $\sum_{t=1}^{K} \pi_{t} Q_{t}$ modify this DMU by applying trade-offs $\left(P_{t}, Q_{t}\right)$ in some proportions $\pi_{t} \geq 0$. The resulting DMU is further modified by increasing its inputs by the vector $d$ and by reducing its outputs by the vector $e$, which is consistent with the assumption of free disposability. Provided all inputs and outputs remain nonnegative, the DMU $(X, Y)$ on the right-hand side of (4) is considered a member of technology $\mathcal{T}_{\text {CRS-TO }} \cdot{ }^{4}$

The VRS technology $\mathcal{T}_{\text {VRS-TO }}$ is defined similarly, by additionally requiring that vector $\lambda$ satisfies the normalising condition

$$
\sum_{j=1}^{n} \lambda_{j}=1
$$

Remark 1. It is well known that the incorporation of weight restrictions (1) in the multiplier model (2) may result in its infeasibility, which corresponds to the unbounded optimal value of the envelopment program (3). Podinovski and Bouzdine-Chameeva (2013, 2015) show that this happens when weight restrictions generate free production ${ }^{5}$ in the CRS technology $\mathcal{T}_{\text {CRS-TO }}$ and call such weight restrictions inconsistent with the data set. Furthermore, weight restrictions may be inconsistent even if the multiplier and therefore envelopment models (2) and (3) (and their VRS analogues) have a finite optimal value for each observed DMU. Therefore the inconsistency of weight restrictions cannot be detected simply by solving standard models (2) or (3). Podinovski and Bouzdine-Chameeva (2013, 2015) develop special analytical and computational methods for checking if the weight restrictions (1) are consistent. If weight restrictions are inconsistent, this means that we made an error in their specification and that the weight restrictions need reassessing. In our further development we assume that the weight restrictions (1) are consistent - this guarantees that models (2) and (3), their VRS analogues and corresponding input-oriented variants have a finite optimal solution.

\footnotetext{
${ }^{4}$ The CRS technology $\mathcal{T}_{\text {CRS-TO }}$ and the corresponding VRS technology $\mathcal{T}_{\text {VRS-TO }}$ can also be defined axiomatically (Podinovski, 2004a).

${ }^{5}$ Technology $\mathcal{T}$ allows free production if there exists a nonzero vector of outputs $Y$ that can be produced from a zero vector of inputs, i.e., $(0, Y) \in \mathcal{T}$.
} 


\section{Examples}

The following examples show that the single optimisation stage applied to DEA models with weight restrictions may lead to the identification of “efficient targets” with negative inputs.

\section{$<$ Table 1 here $>$}

Example 1. Table 1 shows DMUs $A$ and $B$ evaluated on two inputs and one output. Suppose we wish to assess the output radial efficiency of DMU $B$ using the single-stage VRS DEA model, in which $\varepsilon=0.001 .^{6}$ Further assume that we wish to incorporate the following weight restriction that links the weights $v_{1}$ and $v_{2}$ of the first and second inputs:

$$
-2 v_{1}+1 v_{2} \geq 0
$$

With weight restriction (6), the conventional single-stage output-oriented multiplier VRS model for DMU $B$ is stated as follows:

$$
\min 1 v_{1}+3 v_{2}+\omega
$$

subject to

$$
\begin{aligned}
& 1 u_{1}=1, \\
& 3 v_{1}+1 v_{2}-1 u_{1}+\omega \geq 0, \\
& 1 v_{1}+3 v_{2}-1 u_{1}+\omega \geq 0, \\
& -2 v_{1}+1 v_{2} \geq 0, \\
& v_{1}, v_{2}, u_{1} \geq 0.001, \omega \text { sign free. }
\end{aligned}
$$

As discussed in Section 2, the weight restriction (6) generates the dual production trade-off stated as $P_{1}=(-2,1)^{\top} \in \mathbb{R}^{2}$ and $Q_{1}=(0) \in \mathbb{R}^{1}$. The envelopment model dual to (7) takes on the following form:

$$
\max \eta+0.001\left(d_{1}+d_{2}+e_{1}\right)
$$

subject to

$$
\begin{aligned}
& 3 \lambda_{A}+1 \lambda_{B}-2 \pi_{1}+1 d_{1}=1, \\
& 1 \lambda_{A}+3 \lambda_{B}+1 \pi_{1}+1 d_{2}=3, \\
& 1 \lambda_{A}+1 \lambda_{B}-1 e_{1}=1 \eta, \\
& \lambda_{A}+\lambda_{B}=1,
\end{aligned}
$$

\footnotetext{
${ }^{6}$ Taking smaller values of $\varepsilon$, e.g., $\varepsilon=10^{-6}$, makes no difference to our example and leads to the same effect.
} 


$$
\lambda_{A}, \lambda_{B}, \pi_{1}, d_{1}, d_{2}, e_{1} \geq 0, \eta \text { sign free. }
$$

The above envelopment program has a unique exact optimal solution (this is seen from the graph discussed below and can be proved formally using duality theory ${ }^{7}$ ):

$$
\hat{\lambda}_{\mathrm{A}}=1, \hat{\lambda}_{\mathrm{B}}=0, \hat{\pi}_{1}=2, \hat{d}_{1}=2, \hat{d}_{2}=0, \hat{e}_{1}=0, \hat{\eta}=1 \text {. }
$$

This solution does not appear problematic: one would conventionally interpret the reciprocal value $1 / \hat{\eta}=1$ as the output radial efficiency of DMU $B$ and correctly conclude that this DMU is output radial efficient. (Note that, in this example, 1 is the exact output radial efficiency of DMU $B$ - it is obtained with $\varepsilon=0$.) Furthermore, the non-zero optimal slack $\hat{d}_{1}=2$ appears to indicate the amount of mix inefficiency that DMU $B$ should eliminate by reducing Input 1 , in order to become fully efficient.

A problem with the above solution arises if we calculate the efficient target to DMU $B$. As in the case without weight restrictions, we define the efficient target as the radial target of DMU $B$ modified by the optimal slacks. ${ }^{8}$ This gives us the following target DMU $(\hat{X}, \hat{Y})$ in which the first two components are inputs and the last is output:

$$
\left(\begin{array}{l}
\hat{X} \\
\hat{Y}
\end{array}\right)=\left(\begin{array}{l}
1-1 \hat{d}_{1} \\
3-1 \hat{d}_{2} \\
1 \hat{\eta}+1 \hat{e}_{1}
\end{array}\right)=\left(\begin{array}{l}
-1 \\
3 \\
1
\end{array}\right) .
$$

\footnotetext{
${ }^{7}$ Indeed, consider dual programs (7) and (8). Note that $\Gamma=\left\langle v_{1}, v_{2}, u_{1}, \omega\right\rangle$, where $v_{1}=0.001, v_{2}=0.002, u_{1}=1$ and $\omega=0.995$ (all values are exact), is feasible in program (7). (Solution $\Gamma$ was obtained by a linear solver but its optimality is verified below by duality theory.) Furthermore, the objective functions of programs (7) and (8) calculated at the respective feasible solutions $\Gamma$ and (9) are equal to the same value 1.002. By duality, solution $\Gamma$ is optimal in program (7), and solution (9) is optimal in program (8). Substituting $\Gamma$ into the constraints (7) and using complementary slackness conditions, we conclude that, in any optimal solution to (8), we have $\lambda_{B}=d_{2}=e_{1}=0$. Substituting these values into the constraints of (8), we prove that optimal solution (9) is unique.

${ }^{8}$ If the resulting inputs and outputs are nonnegative, this target DMU is by definition a member of the expanded technology $\mathcal{T}_{\text {VRS-TO }}$. Because Input 1 of DMU $(\hat{X}, \hat{Y})$ in formula (10) is negative, $(\hat{X}, \hat{Y}) \notin \mathcal{T}_{\text {VRS-TO }}$. An alternative definition is given by Allen et al. (1997) according to which the efficient target is defined as the combination of observed DMUs taken with the optimal coefficients $\lambda_{j}$. For this definition, the target DMU lies in the original technology (unmodified by weight restrictions), and no problems highlighted by our example arise. However, as noted by Allen et al. (1997), in the latter definition the radial nature of efficiency breaks down and some of the target inputs and outputs may be worse than their current levels observed at DMU $\left(X_{o}, Y_{o}\right)$. Thus, in our example, according to the latter definition, the efficient target of DMU $B$ is DMU $A$ (whose $\hat{\lambda}_{A}$ is equal to 1 ). Note that Input 1 of DMU $A$ is worse (greater) than its value at DMU $B$. It is also worth noting that, in the case of CRS, it is theoretically possible (at least in carefully constructed examples) that all optimal coefficients $\lambda_{j}$ are equal to zero. Then, calculating the efficient target based only on the optimal vector $\lambda$, we obtain the zero DMU (the origin) as the efficient target, which clearly appears problematic.
} 
It is clear that the exact optimal solution to the single-stage program (8) results in an inappropriate "efficient target" with a negative value of the first input, and DMU $(\hat{X}, \hat{Y})$ is not in technology $\mathcal{T}_{\text {VRS-TO }}$.

Figure 1 illustrates the above solution. The darker area above and to the right of the line $K B A L$ is the standard VRS technology in the two input dimensions. (In this example the output dimension is not important and is not shown.) As seen from model (8) and according to Definition 1, the DMUs in the VRS technology are further modified by the trade-off $P_{1}=(-2,1)^{\top}, Q_{1}=(0)$ generated by the weight restriction (6) and used in a variable proportion $\pi_{1} \geq 0$. For example, the application of trade-off $\left(P_{1}, Q_{1}\right)$ to DMU $A$ with $\pi_{1}=1$ adds hypothetical DMU $F$ to the technology. The same trade-off with $\pi_{1}=1.5$ creates DMU C. Overall, letting $\pi_{1}$ to change in the range $[0,1.5]$ generates the line $C A$. By the assumption of free disposability of inputs, the effect of this trade-off is the addition of the light grey area above the line $C A$ to the original VRS technology. Note that we cannot take $\pi_{1}>1.5$ because this results in negative values of Input 1 . In particular, DMU $D$ is not included in the technology $\mathcal{T}_{\text {VRS-TO }}$. Also note that the application of trade-off $\left(P_{1}, Q_{1}\right)$ to the other DMUs (e.g., to DMU $B$ ) does not add new points to the technology.

The incorrect target DMU $(\hat{X}, \hat{Y})$ corresponds to point $D$ in Figure 1 and is outside the technology.

It may appear reasonable to correct this problem by replacing the negative "target" input -1 in (10) by zero and taking DMU $(\tilde{X}, \tilde{Y})=(0,3,1)$ as the "corrected" target unit. The latter DMU corresponds to point $G$ in Figure 1. It is, however, clear that $G$ is dominated by unit $C$ and is therefore inefficient and cannot be considered an efficient target for $B$. This shows that replacing negative inputs by zeros is theoretically unsound.

The above example demonstrates that the incorporation of a secondary goal of maximising input and output slacks as in program (8) may lead to negative input target values. This is consistent with the finding of Podinovski (2007b) who shows that the simple maximisation of the input and output slacks in a separate optimisation stage in programs with weight restrictions may also lead to negative inputs values. Note that this problem does not arise in standard CRS and VRS DEA models without weight restrictions, with a small lower bound $\varepsilon>0$ on the input and output weights. 
Example 2. In the above example DMU $B$ is output radial efficient. A minor modification of this example shows that the same negative input targets may be obtained for a DMU that is output radial inefficient. Indeed, consider DMU $B^{\prime}$ that has the same two inputs as DMU $B$ in Table 1 and the output equal to 0.5. Assume that DMUs $A$ and $B^{\prime}$ are observed. Solving the model similar to (8), we obtain the optimal solution (9) in which $\hat{\eta}=1$ is replaced by $\hat{\eta}=2$. This means that the output radial efficiency of DMU $B^{\prime}$ is equal to $1 / \hat{\eta}=0.5$. Its "efficient target” is the same as above and defined by formula (10). In this modified example, point $B$ in Figure 1 represents the output radial projection of DMU $B^{\prime}$.

Example 3. Negative input targets may also occur in an input-oriented model. Consider observed DMUs $A$ and $B$ in Table 2. To assess the input radial efficiency of DMU $B$ in a VRS model with weight restriction (6), we solve the following program:

$\min \theta-0.001\left(d_{1}+d_{2}+d_{3}+e_{1}\right)$

subject to

$$
\begin{aligned}
& 3 \lambda_{A}+2 \lambda_{B}-2 \pi_{1}+1 d_{1}=2 \theta, \\
& 1 \lambda_{A}+6 \lambda_{B}+1 \pi_{1}+1 d_{2}=6 \theta, \\
& 1 \lambda_{A}+2 \lambda_{B}+1 d_{3}=2 \theta, \\
& 1 \lambda_{A}+1 \lambda_{B}-1 e_{1}=1, \\
& \lambda_{A}+\lambda_{B}=1, \\
& \lambda_{A}, \lambda_{B}, \pi_{1}, d_{1}, d_{2}, d_{3}, e_{1} \geq 0, \theta \text { sign free. }
\end{aligned}
$$

It is straightforward to verify that the unique exact optimal solution to program (11) is

$$
\tilde{\lambda}_{A}=1, \tilde{\lambda}_{B}=0, \tilde{\pi}_{1}=2, \tilde{d}_{1}=2, \tilde{d}_{2}=0, \tilde{d}_{3}=0, \tilde{e}_{1}=0, \tilde{\theta}=0.5 .
$$

Therefore, the input radial efficiency of DMU $B$ is equal to 0.5. Repeating computations as in Example 1, we obtain the "efficient target" of DMU B with a negative input:

$$
\left(\begin{array}{c}
\tilde{X} \\
\tilde{Y}
\end{array}\right)=\left(\begin{array}{l}
2 \tilde{\theta}-1 \tilde{d}_{1} \\
6 \tilde{\theta}-1 \tilde{d}_{2} \\
2 \tilde{\theta}-1 \tilde{d}_{3} \\
1+1 \tilde{e}_{1}
\end{array}\right)=\left(\begin{array}{l}
-1 \\
3 \\
1 \\
1
\end{array}\right)
$$

Example 4. Similar examples can be given for the case of CRS. Consider the data set in Table 2. To assess the input radial efficiency of DMU $B$, we solve program (11) from which 
we remove the normalising equality $\lambda_{A}+\lambda_{B}=1$. This program has the same unique optimal solution (12) as in the case of VRS, resulting in the same "efficient target" (13) with a negative input. The CRS input radial efficiency of DMU $B$ is equal to 0.5.

Similarly, the CRS output radial efficiency of DMU $B$ is, as expected, also equal to 0.5. In this case, the target unit for DMU $B$ is $\left(X^{\prime}, Y^{\prime}\right)=(-2,6,2,2)$, where the first three components are the inputs and the fourth is the output. Note that the target value for Input 1 is negative.

\section{The corrected single-stage model}

Based on Definition 1, below we suggest a simple modification to the single-stage envelopment and multiplier models that overcome the problem highlighted in Section 3. These models identify efficient projections of inefficient units on the boundary of technologies $\mathcal{T}_{\text {CRS-TO }}$ and $\mathcal{T}_{\text {VRS-TO }}$. In particular, by definition of such technologies, this implies the nonnegativity of the target inputs and outputs.

\subsection{Output-oriented models}

Taking into account Definition 1, the single-stage output-oriented program that identifies an efficient target within the technology $\mathcal{T}_{\text {CRS-TO }}$ may be stated in the following form: ${ }^{9}$

$$
\max \eta+\varepsilon\left(\sum_{i=1}^{m} \xi_{i}+\sum_{r=1}^{s} \varsigma_{r}\right)
$$

subject to $\left(X_{o}-\xi, \eta Y_{o}+\varsigma\right) \in \mathcal{T}_{C R S-T O}, \xi, \varsigma \geq 0$.

The full statement of program (14) is as follows:

$$
\begin{aligned}
\max \eta+\varepsilon( & \left.\sum_{i=1}^{m} \xi_{i}+\sum_{r=1}^{s} \varsigma_{r}\right) \\
\text { subject to } & \sum_{j=1}^{n} \lambda_{j} X_{j}+\sum_{t=1}^{K} \pi_{t} P_{t}+d=X_{o}-\xi, \\
& \sum_{j=1}^{n} \lambda_{j} Y_{j}+\sum_{t=1}^{K} \pi_{t} Q_{t}-e=\eta Y_{o}+\varsigma, \\
& X_{o}-\xi \geq 0,
\end{aligned}
$$

\footnotetext{
${ }^{9}$ To avoid confusion with the vectors $e$ and $d$ used in Definition 1 , we denote the input slacks $\xi \in \mathbb{R}_{+}^{m}$ and output slacks $\varsigma \in \mathbb{R}_{+}^{s}$.
} 


$$
\begin{aligned}
& \eta Y_{o}+\varsigma \geq 0, \\
& \lambda, \pi, d, e, \xi, \varsigma \geq 0, \eta \text { sign free. }
\end{aligned}
$$

Note that program (15) can be simplified. First, at any of its optimal solutions, vector $e$ must be a zero vector. ${ }^{10}$ Second, for all sufficiently small $\varepsilon>0$ condition (15e) is redundant. ${ }^{11}$ Therefore, program (15) can be restated as follows:

$$
\begin{aligned}
\max \eta+\varepsilon\left(\sum_{i=1}^{m} \xi_{i}+\sum_{r=1}^{s} \varsigma_{r}\right) & \\
\text { subject to } \quad & \sum_{j=1}^{n} \lambda_{j} X_{j}+\sum_{t=1}^{K} \pi_{t} P_{t}+d=X_{o}-\xi, \\
& \sum_{j=1}^{n} \lambda_{j} Y_{j}+\sum_{t=1}^{K} \pi_{t} Q_{t}=\eta Y_{o}+\varsigma, \\
& X_{o}-\xi \geq 0, \\
& \lambda, \pi, d, \xi, \varsigma \geq 0, \eta \text { sign free. }
\end{aligned}
$$

Let $\left\langle\lambda^{*}, \pi^{*}, d^{*}, \xi^{*}, \varsigma^{*}, \eta^{*}\right\rangle$ be an optimal solution to program (16). Define the target DMU as

$$
\left(X^{*}, Y^{*}\right)=\left(X_{o}-\xi^{*}, \eta^{*} Y_{o}+\varsigma^{*}\right)
$$

Because $\left(X^{*}, Y^{*}\right)$ satisfies equations (4) (where $e$ is a zero vector), and $X^{*} \geq 0$ and $Y^{*} \geq 0$, we have $\left(X^{*}, Y^{*}\right) \in \mathcal{T}_{\text {CRS-TO }}$. The next result shows that DMU $\left(X^{*}, Y^{*}\right)$ can be regarded as an efficient target of DMU $\left(X_{o}, Y_{o}\right)$.

Theorem 1. DMU $\left(X^{*}, Y^{*}\right)$ is efficient in technology $\mathcal{T}_{\text {CRS-TO }}$.

A proof of Theorem 1 is given in Appendix A.

Denoting $v, u$ and $w$ the dual vectors corresponding to constraints (16b) and negated constraints (16c) and (16d), respectively, the dual to program (16) can be stated as follows: ${ }^{12}$

$$
\min \left(v^{\top}+w^{\top}\right) X_{o}
$$

\footnotetext{
10 Indeed, if we assume to the contrary that $e \geq 0$ and $e \neq 0$, then redefining $\tilde{e}=0$ and $\tilde{\varsigma}=\varsigma+e$ keeps (15c) and (15e) true, while improving the objective function (15a), which is impossible due to the assumed optimality of the current solution. Therefore, vector $e$ in program (15) can be assumed zero and removed from the formulation.

${ }^{11}$ Theorem 2.1 proved in Sherali (1982) implies that, for all sufficiently small $\varepsilon>0$, an optimal $\eta$ in program (15) is also optimal in program (3) and is, therefore, nonnegative. Because vector $\varsigma$ is nonnegative, for all sufficiently small $\varepsilon>0$, constraint (15e) is satisfied at any optimal solution to (15) and can be omitted.

${ }^{12}$ The nonnegativity condition on vector $v$ in program (18) is dual to the primal vector $d \geq 0$.
} 
subject to $\quad u^{\top} Y_{o}=1$,

$$
\begin{array}{ll}
v^{\top} X_{j}-u^{\top} Y_{j} \geq 0, & j=1, \ldots, n, \\
v^{\top} P_{t}-u^{\top} Q_{t} \geq 0, & t=1, \ldots, K, \\
v_{i}+w_{i} \geq \varepsilon, & i=1, \ldots, m, \\
u_{r} \geq \varepsilon, & r=1, \ldots, s, \\
v, w \geq 0 . &
\end{array}
$$

It is worth noting that the above program is different to model (7) which was shown to be generally incorrect by an example in Section 3 .

\subsection{Input-oriented models}

In the input orientation, program (16) needs an obvious modification as follows, where $\theta$ represents the proportional input improvement factor:

$$
\begin{aligned}
& \min \theta-\varepsilon\left(\sum_{i=1}^{m} \xi_{i}+\sum_{r=1}^{s} \varsigma_{r}\right) \\
& \text { subject to } \quad \sum_{j=1}^{n} \lambda_{j} X_{j}+\sum_{t=1}^{K} \pi_{t} P_{t}+d=\theta X_{o}-\xi, \\
& \sum_{j=1}^{n} \lambda_{j} Y_{j}+\sum_{t=1}^{K} \pi_{t} Q_{t}=Y_{o}+\varsigma, \\
& \theta X_{o}-\xi \geq 0, \\
& \lambda, \pi, d, \xi, \varsigma \geq 0, \theta \text { sign free. }
\end{aligned}
$$

Any optimal solution to program (19) defines an efficient target to DMU $\left(X_{o}, Y_{o}\right)$ in the usual way:

$$
\left(X^{*}, Y^{*}\right)=\left(\theta^{*} X_{o}-\xi^{*}, Y_{o}+\varsigma^{*}\right),
$$

where $\theta^{*}, \xi^{*}$ and $\varsigma^{*}$ are components of an optimal solution to (19). It is straightforward to verify that Theorem 1 remains true in this case.

Using similar notation for dual vectors $v, u$ and $w$ as above, the dual to program (19) is stated as follows:

$$
\max u^{\top} Y_{o}
$$

subject to $\quad\left(v^{\top}+w^{\top}\right) X_{o}=1$,

$$
v^{\top} X_{j}-u^{\top} Y_{j} \geq 0, \quad j=1, \ldots, n,
$$




$$
\begin{array}{ll}
v^{\top} P_{t}-u^{\top} Q_{t} \geq 0, & t=1, \ldots, K, \\
v_{i}+w_{i} \geq \varepsilon, & i=1, \ldots, m, \\
u_{r} \geq \varepsilon, & r=1, \ldots, s, \\
v, w \geq 0 . &
\end{array}
$$

Remark 2. In the case of VRS, the envelopment program (16) includes an additional normalising equality (5). The corresponding multiplier program (18) is modified accordingly, by incorporating the additional sign-free variable $\omega$ corresponding to (5). The inputorientated program (19) and its dual (20) are treated in the same way. An obvious analogue of Theorem 1 remains valid in all these cases.

\section{Revisiting the solution of Example 1}

Below we illustrate the use of the new models by revisiting Example 1. As demonstrated, solving the single-stage model (8) leads to an incorrect target $(\hat{X}, \hat{Y})$ for the inefficient DMU $B$. As shown above, instead of (8), we should solve program (16) which takes on the following form:

$\max \eta+0.001\left(\xi_{1}+\xi_{2}+\varsigma_{1}\right)$

subject to

$$
\begin{aligned}
& 3 \lambda_{A}+1 \lambda_{B}-2 \pi_{1}+1 d_{1}=1-\xi_{1}, \\
& 1 \lambda_{A}+3 \lambda_{B}+1 \pi_{1}+1 d_{2}=3-\xi_{2}, \\
& 1 \lambda_{A}+1 \lambda_{B}=1 \eta+\varsigma_{1}, \\
& 1-\xi_{1} \geq 0 \\
& 3-\xi_{2} \geq 0 \\
& \lambda_{A}+\lambda_{B}=1, \\
& \lambda_{A}, \lambda_{B}, \pi_{1}, d_{1}, d_{2}, \xi_{1}, \xi_{2}, \varsigma_{1} \geq 0, \eta \text { sign free. }
\end{aligned}
$$

It is straightforward to verify that (21) has the following exact unique optimal solution:

$$
\lambda_{A}^{*}=1, \lambda_{B}^{*}=0, \pi_{1}^{*}=1.5, d_{1}^{*}=d_{2}^{*}=0, \xi_{1}^{*}=1, \xi_{2}^{*}=0.5, \varsigma_{1}^{*}=0, \eta^{*}=1
$$

The corresponding efficient target is stated as follows: 


$$
\left(\begin{array}{l}
X^{*} \\
Y^{*}
\end{array}\right)=\left(\begin{array}{l}
1-\xi_{1}^{*} \\
3-\xi_{2}^{*} \\
1 \eta^{*}+\varsigma_{1}^{*}
\end{array}\right)=\left(\begin{array}{l}
0 \\
2.5 \\
1
\end{array}\right) .
$$

As discussed above, by the construction of program (16), we have $\left(X^{*}, Y^{*}\right) \in \mathcal{T}_{\text {VRS-TO }}$. By Theorem 1, DMU $\left(X^{*}, Y^{*}\right)$ is efficient. In Figure 1, this DMU corresponds to point $C$. Note that DMU $\left(X^{*}, Y^{*}\right)$ dominates $(\tilde{X}, \tilde{Y})=(0,3,1)$ obtained in Section 3 by replacing the negative input in unit (10) by zero.

\section{Conclusion}

The identification of efficient targets of inefficient DMUs in conventional CRS and VRS DEA models may be viewed as a combination of two stages. According to Ali and Seiford (1993b), the first stage identifies the input or output radial projection of the DMU under the assessment on the boundary of the technology. The second stage aims at the elimination of any mix inefficiency at the radial target DMU. This is achieved by the maximisation of the sum of residual input and output slacks.

Alternative single-stage multiplier DEA models incorporate a small (theoretically infinitesimal) lower bound $\varepsilon>0$ on the input and output weights. Their dual envelopment models simultaneously assess the input or output radial efficiency of the DMU as the primary goal, and also account for the input and output slacks in the objective function, as a secondary goal.

It is well known that practical solutions of single-stage DEA models with a very small finite $\varepsilon$ may cause unacceptable computational inaccuracies (Ali and Seiford, 1993a). Solving such programs generally requires that the tolerance parameters of the simplex method be specifically fine-tuned to the data set. Despite these computational shortcomings, stating DEA models as single-stage programs is very common in the DEA literature.

In our paper we examine DEA models with additional weight restrictions, which is a common technique used in many applications. In envelopment DEA models, weight restrictions take on the form of production trade-offs between inputs and outputs. Podinovski (2007b) shows that such models can be solved by the two-stage method of Ali and Seiford (1993b) in which the second stage needs a modification. 
The current paper looks at the single-stage solutions of DEA models with weight restrictions. This is motivated by a large DEA literature in which such models are stated simultaneously with the lower bound $\varepsilon$ on the input and output weights.

The examples given in our paper show that the single-stage VRS and CRS DEA models with weight restrictions, even if solved exactly and for an arbitrarily small $\varepsilon>0$, may result in negative inputs of the target DMUs. We show that this problem arises because not all conditions of the definition of technology with weight restrictions are properly accounted for in standard DEA formulations. We subsequently develop a single-stage DEA model with weight restrictions that theoretically produces efficient targets located on the boundary of the assumed production technology. We illustrate the use of this model by an example in which we obtain its exact solution and illustrate it by a graph.

Similar to conventional DEA models without weight restrictions, the suggested single-stage models might still be problematic in practical computations (although all computer solutions of the examples given in our paper turned out to be exact with the default parameters of the simplex method, as verified by duality theory). Therefore in practice, similar to DEA models without weight restrictions, it might still be more straightforward to perform computations in separate stages. 


\section{Appendix A: Proof of Theorem 1}

Suppose $\left(X^{*}, Y^{*}\right)$ defined by (17) is inefficient in $\mathcal{T}_{C R S-T O}$. Then there exists a DMU $\left(X^{\prime}, Y^{\prime}\right) \in \mathcal{T}_{C R S-T O}$ such that $X^{\prime}=X^{*}-\delta$ and $Y^{\prime}=Y^{*}+\gamma$, where at least one of the vectors $\delta \in \mathbb{R}_{+}^{m}$ and $\gamma \in \mathbb{R}_{+}^{s}$ is not a zero vector. Because DMU $\left(X^{\prime}, Y^{\prime}\right) \in \mathcal{T}_{C R S-T O}$, it satisfies (4) with some vectors $\lambda^{\prime}, \pi^{\prime}, d^{\prime}, e^{\prime} \geq 0$. Then

$$
\begin{aligned}
& \sum_{j=1}^{n} \lambda_{j}^{\prime} X_{j}+\sum_{t=1}^{K} \pi_{t}^{\prime} P_{t}+d^{\prime}=X^{\prime}=X_{o}-\xi^{*}-\delta, \\
& \sum_{j=1}^{n} \lambda_{j}^{\prime} Y_{j}+\sum_{t=1}^{K} \pi_{t}^{\prime} Q_{t}-e^{\prime}=Y^{\prime}=\eta^{*} Y_{o}+\varsigma^{*}+\gamma .
\end{aligned}
$$

Denote $\tilde{\xi}=\xi^{*}+\delta$ and $\tilde{\varsigma}=\varsigma^{*}+\gamma+e^{\prime}$. Then $\left\langle\lambda^{\prime}, \pi^{\prime}, d^{\prime}, \tilde{\xi}, \tilde{\zeta}, \eta^{*}\right\rangle$ is a feasible solution of program (16). Note that

$$
\sum_{i=1}^{m} \tilde{\xi}_{i}+\sum_{r=1}^{s} \tilde{\zeta}_{r}>\sum_{i=1}^{m} \xi_{i}^{*}+\sum_{r=1}^{s} \varsigma_{r}^{*} .
$$

Therefore the solution $\left\langle\lambda^{*}, \pi^{*}, d^{*}, \xi^{*}, \varsigma^{*}, \eta^{*}\right\rangle$ is not optimal in program (16), which contradicts the assumption. Therefore $\left(X^{*}, Y^{*}\right)$ is efficient. 


\section{References}

Allen, R., Athanassopoulos. A., Dyson, R.G., Thanassoulis, E. (1997). Weights restrictions and value judgements in data envelopment analysis: Evolution, development and future directions. Annals of Operations Research, 73, 13-34.

Ali, A.I., Seiford, L.M. (1993a). Computational accuracy and infinitesimals in data envelopment analysis. INFOR, 31, 290-297.

Ali, A.I., Seiford, L.M. (1993b). The mathematical programming approach to efficiency analysis. In H.O. Fried, C.A.K. Lovell, S.S. Schmidt (Eds.), The measurement of productive efficiency: Techniques and applications (pp. 120-159). New York: Oxford University Press.

Amado, C.A.F., Dyson, R.G. (2009). Exploring the use of DEA for formative evaluation in primary diabetes care: An application to compare English practices. Journal of the Operational Research Society, 60, 1469-1482.

Amado, C.A.F., Santos, S.P. (2009) Challenges for performance assessment and improvement in primary health care: The case of the Portuguese health centres. Health Policy, 91, 43-56.

Atici, K.B., Podinovski, V.V. (2015). Using data envelopment analysis for the assessment of technical efficiency of units with different specialisations: an application to agriculture. Omega, 54, 72-83.

Banker, R.D., Charnes, A., Cooper, W.W. (1984). Some models for estimating technical and scale inefficiencies in data envelopment analysis. Management Science, 30, 10781092.

Charnes, A., Cooper, W.W., Rhodes, E. (1978). Measuring the efficiency of decision making units. European Journal of Operational Research, 2, 429-444.

Charnes, A., Cooper, W.W., Wei, Q.L., Huang, Z.M. (1989). Cone ratio data envelopment analysis and multi-objective programming. International Journal of Systems Science, 20, 1099-1118.

Cook, W.D., Zhu, J. (2008). Context-dependent assurance regions in DEA. Operations Research, 56, 69-78.

Cooper, W.W., Seiford, L.M., Tone, K. (2007). Data envelopment analysis. A comprehensive text with models, applications, references and DEA-Solver software (2nd ed.). New York: Springer Science + Business Media.

Dyson, R.D., Thanassoulis, E. (1988). Reducing weight flexibility in data envelopment analysis. Journal of the Operational Research Society, 39, 563-576. 
Podinovski, V.V. (1999). Side effects of absolute weight bounds in DEA models. European Journal of Operational Research, 115, 583-595.

Podinovski, V.V. (2004a). Production trade-offs and weight restrictions in data envelopment analysis. Journal of the Operational Research Society, 55, 1311-1322.

Podinovski, V.V. (2004b). Suitability and redundancy of non-homogeneous weight restrictions for measuring the relative efficiency in DEA. European Journal of Operational Research, 154, 380-395.

Podinovski, V.V. (2005). The explicit role of weight bounds in models of data envelopment analysis. Journal of the Operational Research Society, 56, 1408-1418.

Podinovski, V.V. (2007a). Improving data envelopment analysis by the use of production trade-offs. Journal of the Operational Research Society, 58, 1261-1270.

Podinovski, V.V. (2007b). Computation of efficient targets in DEA models with production trade-offs and weight restrictions. European Journal of Operational Research, 181, 586-591.

Podinovski, V.V. (2015) DEA models with production trade-offs and weight restrictions. In J. Zhu (Ed.), Data envelopment analysis: A handbook of models and methods (pp. 105-144). New York: Springer Science+Business Media.

Podinovski, V.V., Bouzdine-Chameeva, T. (2013). Weight restrictions and free production in data envelopment analysis. Operations Research, 61, 426-437.

Podinovski, V.V., Bouzdine-Chameeva, T. (2015). Consistent weight restrictions in data envelopment analysis. European Journal of Operational Research, 244, 201-209.

Roll, Y., Cook, W.D., Golany, B. (1991). Controlling factor weights in data envelopment analysis. IIE Transactions, 23, 2-9.

Santos, S.P., Amado, C.A.F., Rosado, J.R. (2011). Formative evaluation of electricity distribution utilities using data envelopment analysis. Journal of the Operational Research Society, 62, 1298-1319.

Santos, S.P., Amado, C.A.F. (2014). On the need for reform of the Portuguese judicial system - does data envelopment analysis assessment support it? Omega, 47, 1-16.

Sherali, H.D. (1982) Equivalent weights for lexicographic multi-objective programs: Characterizations and computations. European Journal of Operational Research, 11, 367-379.

Thanassoulis, E., Portela, M.C., Allen, R. (2004). Incorporating value judgements in DEA. In W.W. Cooper, L.M. Seiford, J. Zhu (Eds.), Handbook on data envelopment analysis (pp. 99-138). Boston: Kluwer Academic Publishers. 
Thanassoulis, E., Portela, M.C.S., Despić, O. (2008). Data envelopment analysis: The mathematical programming approach to efficiency analysis. In H.O. Fried, C.A.K. Lovell, S.S. Schmidt (Eds.), The measurement of productive efficiency and productivity growth (pp. 251-420). New York: Oxford University Press. 


\begin{tabular}{|c|c|c|c|}
\hline DMU & Input 1 & Input 2 & Output \\
\hline$A$ & 3 & 1 & 1 \\
\hline$B$ & 1 & 3 & 1 \\
\hline
\end{tabular}

Table 1 DMUs $A$ and $B$ in Example 1.

\begin{tabular}{|c|c|c|c|c|}
\hline DMU & Input 1 & Input 2 & Input 3 & Output \\
\hline$A$ & 3 & 1 & 1 & 1 \\
\hline$B$ & 2 & 6 & 2 & 1 \\
\hline
\end{tabular}

Table 2 DMUs $A$ and $B$ in Examples 3 and 4. 


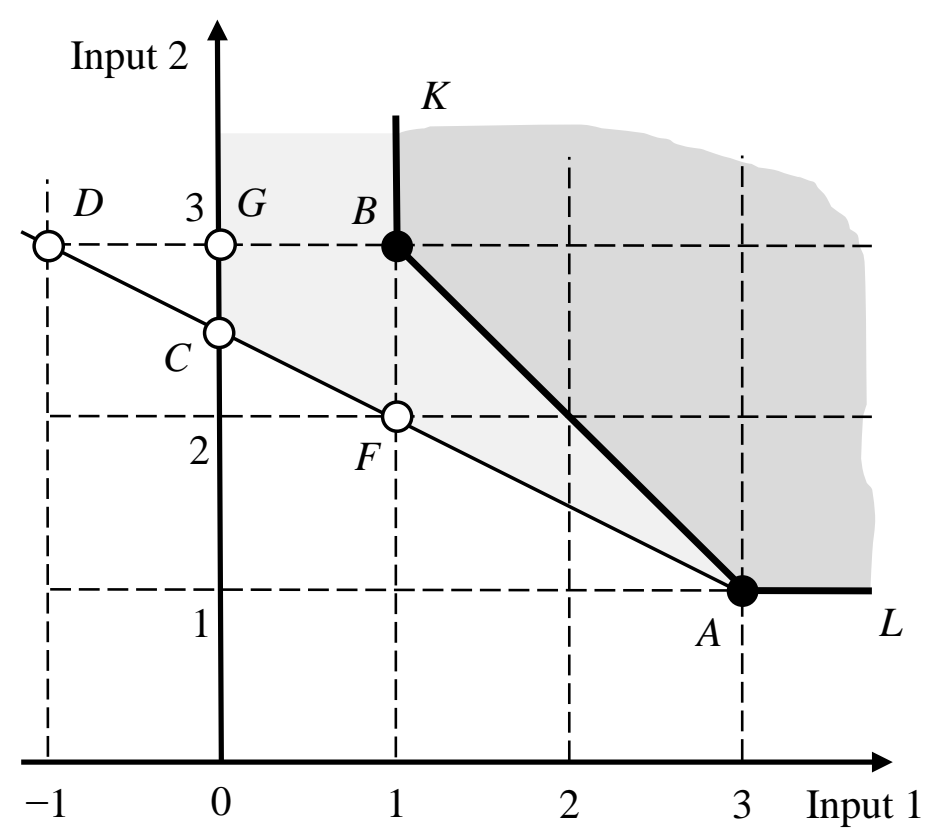

Figure 1 The VRS technology induced by DMUs $A$ and $B$ in Example 1 (shown in two input dimensions). The line $A C$ corresponds to weight restriction (6). 